\section{Estimativa de subnotificação de casos de aids em uma capital do Nordeste}

\section{Estimation of underreporting of AIDS cases in a Brazilian Northeast metropolis}

\section{Valéria Freire Gonçalves ${ }^{1,2}$}

\section{Lígia Regina Franco Sansigolo Kerr ${ }^{2}$}

Rosa Maria Salani Mota ${ }^{3}$

\section{João Maurício Araújo Mota ${ }^{3}$}

${ }^{1}$ Núcleo de Epidemiologia da Secretaria da Saúde do Estado do Ceará.

${ }^{2}$ Departamento de Saúde Comunitária da Universidade Federal do Ceará.

${ }^{3}$ Departamento de Estatistica e Matemática Aplicada da Universidade Federal do Ceará.

Auxílio financeiro: Fundação Cearense de Apoio ao Desenvolvimento Científico e Tecnológico FUNCAP/Processo №. 0578/06

Aprovado pelo Comitê de Ética em Pesquisa da Universidade Federal do Ceará.

Correspondência: Valéria Freire Gonçalves. Rua Paulo Morais, 175 apto 501 - Papicu, Fortaleza,

Ceará - CEP 60.175-175. E-mail: val@saude.ce.gov.br

\section{Resumo}

A subnotificação de casos de Aids representa um dos principais problemas da vigilância epidemiológica da Aids. Este trabalho teve como objetivo conhecer a subnotificação dos casos de Aids em adultos, no município de Fortaleza, nos anos de 2002 e 2003. Estimou-se ainda a subnotificação em dois hospitais de referência, Hospital São José - HSJ e Hospital Geral de Fortaleza - HGF. Utilizou-se o método de captura e recaptura com base nos dados secundários do Sistema de Informação de Agravos de Notificação Sinan, Sistema de Controle de Exames Laboratoriais - Siscel e Sistema de Informações sobre Mortalidade-SIM. Os três sistemas foram comparados dois a dois e considerados como notificados os casos confirmados no Sinan. Os casos foram emparelhados no Programa RecLink II. Após este processo, foram selecionados os casos elegíveis para aplicação do método de captura e recaptura, com a utilização dos estimadores de Lincoln-Petersen e o de Chapman. A subnotificação estimada para Fortaleza foi de $33,1 \%$ e $14,1 \%$, ao se comparar o Sinan com Siscel e SIM, respectivamente. No HSJ e HGF, a subnotificação foi de 5,4\% e 90,5\% na mesma ordem, comparando o Sinan com o Siscel. Este trabalho demonstrou elevada subnotificação de casos de Aids em Fortaleza. A subnotificação, quando avaliada por fonte, isto é, comparando-se o Siscel com o Sinan, é mais de duas vezes superior àquela estimada quando se compara as fontes SIM com Sinan, o que demonstra ser o Siscel uma importante fonte de notificação de casos de Aids. Portanto, recomenda-se que o Sinan-Aids seja comparado, periodicamente, com todas as fontes de informações disponíveis que possam contribuir para a redução da subnotificação de Aids.

Palavras-chave: Síndrome de Imunodeficiência Adquirida. Epidemiologia. Notificação de doenças. Vigilância epidemiológica. Serviços de vigilância epidemiológica. Sistemas de informação. Técnica de estimativa. 


\section{Abstract}

Underreporting is one of the main challenging epidemiologic AIDS surveillance is faced with. The objective of this study was to estimate the level of underreporting of AIDS in adults in Fortaleza in the 2002-2003 period. In addition, the level of underreporting at two referral hospitals (Hospital São José - HSJ, and Hospital Geral de Fortaleza - HGF) was estimated. The study used the capture-recapture method and relied on three secondary databases: SINAN (national disease surveillance), SISCEL (laboratory test control), and SIM (mortality information). SINAN was compared to SISCEL and to SIM. Cases confirmed by SINAN were considered as reported. Cases from the databases were paired using the RecLink II software. Subsequently cases eligible for the capture-recapture method were selected using the Lincoln-Petersen and Chapman estimators. The levels of underreporting were estimated at $33.1 \%$ and $14.1 \%$ for SISCEL and SIM, respectively. Underreporting for SISCEL was $5.4 \%$ at HSJ and $90.5 \%$ at HGF, comparing Siscel to Sinan. The study shows a considerable level of underreporting of AIDS cases in Fortaleza, and suggests that SISCEL is an important source of AIDS reporting considering that it allowed detecting levels of underreporting more than twice the estimates derived from SIM and SINAN databases. Considering the findings of the present study, SINAN-AIDS should be compared periodically with all relevant information systems in order to reduce levels of AIDS underreporting.

Keywords: Acquired Immunodeficiency Syndrome. Epidemiology. Disease reporting. Epidemiological surveillance. Epidemiological surveillance services. Information systems. Estimation technique

\section{Introdução}

No Brasil, a vigilância epidemiológica da Aids tem como propósito principal acompanhar a tendência temporal e espacial da doença, as infecções e os comportamentos de risco, com o objetivo de orientar as ações de prevenção e controle ${ }^{1}$. A vigilância desenvolve suas atividades tendo como referência a notificação universal dos casos de Aids que atendam aos critérios estabelecidos pelo Ministério da Saúde. A evolução das definições dos critérios de Aids acompanha os avanços tecnológicos e a sua disponibilidade ${ }^{2}$. A notificação dos casos de Aids, quando relativamente adequada e com base na história natural da infecção, permite acompanhar, retrospectivamente, $\mathrm{o}$ comportamento da epidemia. É importante, ainda, para validar os dados gerados pelos sistemas de vigilância sentinela e direcionar as atividades de prevenção e planejamento das necessidades de assistência e tratamento da doença ${ }^{3}$.

No entanto, alguns fatores são responsáveis para que seja questionado o número de casos notificados de Aids, tanto qualitativa quanto quantitativamente. Dentre esses fatores, destacam-se a não notificação dos casos pelo profissional médico, em especial os que atendem na rede privada, $\mathrm{o}$ atraso na investigação, além da baixa qualidade da informação coletada que alimenta o Sistema de informação de Agravos de Notificação - Sinan. A coleta muitas vezes despreza os princípios do processo de vigilância epidemiológica e incorpora um olhar burocrático e limitante aos serviços de saúde ${ }^{4-6}$.

A baixa qualidade das investigações pode ser comprovada pelo número de inconsistências encontradas no Sinan, assim como pelo número de informações ignoradas, o que compromete as análises epidemiológicas. Ainda em relação à não notificação dos casos de Aids, um dos fatos que mais contribui é a falta de organização dos sistemas de vigilância epidemiológica, além do estigma que a doença traz aos pacientes ${ }^{5}$. Todos esses fatores reduzem a utilidade da notificação para acompanhamento 
da magnitude e tendência da epidemia.

Em 2004, o Ministério da Saúde, com o objetivo de reduzir a subnotificação e o atraso da notificação dos casos de Aids, estabeleceu a relação entre o banco de dados do Sinan até julho de 2004 e os casos registrados no Sistema de Controle de Exames Laboratoriais - Siscel que apresentavam contagem de linfócitos TCD4+ menor que $350 \mathrm{cel} \mathrm{p} / \mathrm{mm}^{3}$. Dos casos registrados no Siscel, 50,5\% não constavam no Sinan. Para o Ceará, foram identificados $15,0 \%$ dos casos que estavam no Siscel e que ainda não haviam sido notificados no Sinan ${ }^{7}$. Portanto, no Estado do Ceará, apesar dos avanços por melhor qualidade e maior cobertura das informações em saúde e da implementação da vigilância epidemiológica, ainda existe subnotificação significativa dessa doença. Esses resultados reforçam a necessidade de a vigilância epidemiológica utilizar, rotineiramente, outros sistemas de informações complementares.

No Ceará, a curva de incidência da Aids continua ascendente, com 166 (90,2\%) municípios apresentando registros da doença. A situação epidemiológica da Aids no Estado é caracterizada pela pauperização, interiorização, e feminilização dos casos; entretanto, há uma proporção de mais de $50 \%$ de casos ainda na categoria de homens que fazem sexo com homens ${ }^{8}$. No entanto, para direcionar as ações de prevenção e controle, a vigilância epidemiológica deve buscar alternativas para um bom desempenho, reforçando de forma satisfatória a identificação e notificação de casos. A partir das informações obtidas, a vigilância epidemiológica deve concentrar esforços para desenvolver ações efetivas de acordo com a realidade epidemiológica identificada ${ }^{9}$. Dessa forma, este estudo teve como objetivo estimar a subnotificação dos casos de Aids em indivíduos de treze anos de idade ou mais, que residiam em Fortaleza nos anos de 2002 e 2003 e, assim, contribuir para a melhoria do perfil da vigilância desse agravo, a fim de mensurar a magnitude da epidemia e sua tendência, assim como redirecionar as medidas de prevenção e controle.

\section{Metodologia}

\section{Desenho do estudo e população}

Trata-se de um estudo epidemiológico observacional, transversal. A população do estudo foi constituída por todos os casos de aids em indivíduos com treze anos ou mais de idade, residentes no município de Fortaleza diagnosticados nos anos de 2002 e 2003 e que constavam nas fontes pesquisadas, ou seja, no Sinan, Siscel e SIM.

No período de estudo, seis hospitais atendiam casos de aids em Fortaleza. Destes, um atendia ambulatório e internação (Hospital São José - HSJ) e em outro só funcionava o ambulatório (Hospital Geral de Fortaleza - HGF). Estes hospitais eram referências para aids no Estado e concentravam o maior volume de casos da doença. Para estes, estimou-se a subnotificação. Em dois, calculou-se apenas a taxa de subnotificação, pois não havia número de casos suficiente para se proceder as estimativas. Nos demais, não foi possível estimar nenhum desses parâmetros, pois não havia casos registrados no Siscel.

\section{Fonte de dados}

Os dados foram trabalhados a partir de três fontes de informação: Sinan, Siscel e SIM, nas quais o indivíduo com Aids tem a possibilidade de estar registrado. Os dados do Sinan e SIM foram disponibilizados pela Secretaria da Saúde do Estado do Ceará e os do Siscel pelo Ministério da Saúde. O Sinan foi mantido na íntegra com um total de 6.410 casos de Aids notificados, sendo $6.007(93,7 \%)$ confirmados e $403(6,3 \%)$ descartados, considerando-se o período de 1983 até julho de 2005. O objetivo de trabalhar com todos os casos registrados no Sinan foi a possibilidade de identificar casos de Aids encontrados nas fontes Siscel e SIM nos anos de 2002 e 2003, porém já diagnosticados e notificados em anos anteriores ou posteriores aos anos selecionados para o estudo. Portanto, para a aplicação do método de captura e recaptura esses casos 
foram excluídos. Os critérios de inclusão de caso para a fonte Sinan foram a data de diagnóstico nos anos de 2002 e 2003 e o endereço de residência no município de Fortaleza. Desta forma, dos 1.418 casos diagnosticados no Sinan no período de 2002 a 2003, foram excluídos 576 casos com endereço de residência do interior do estado, 142 casos descartados e 12 duplicados, ficando elegíveis para a pesquisa 688 casos. Com relação à fonte Siscel, foram considerados como critério de inclusão os casos com resultado do exame TCD4 $+<350 \mathrm{~mm}^{3} \mathrm{e}$ a data do resultado no Siscel nos anos de 2002 e 2003, e o município de residência. Foram considerados inicialmente 767 casos e destes foram excluídos 271 por ter registro de endereço do interior do estado, 30 casos relativos a menores de 13 anos, 34 casos em que constava endereço do interior, na fonte Sinan, 6 com solicitação de exames de outras unidades federadas e 185 já diagnosticados e notificados no Sinan em anos diferentes de 2002 e 2003. Assim, permaneceram 241 casos elegíveis para a pesquisa. Quanto à fonte SIM, foram selecionados os óbitos ocorridos em 2002 e 2003, cuja causa básica era Aids, conforme o CID-10, cujos códigos variam de B20 a B24. De início foram selecionados os 405 óbitos registrados no Ceará, em 2002 e 2003. Destes foram excluídos 175 com endereço do interior, 2 menores de 13 anos de idade, 8 duplicados, 7 com endereço, no Sinan, do interior do Estado, e 81 óbitos que já estavam notificados no Sinan em anos diferentes de 2002 e 2003. Portanto, foram considerados elegíveis 135 óbitos, sendo 132 óbitos no SIM com endereço de Fortaleza, acrescidos de mais 3 que, embora na Declaração de óbito o endereço fosse do interior, no Sinan constavam como residentes em Fortaleza.

\section{Formação e validação dos bancos de dados}

Para a formação e validação dos bancos de dados, gerou-se um arquivo no Excel com variáveis selecionadas para refinamento e identificação de inconsistências, objeti- vando o preparo dos dados para emparelhamento das fontes. Foram recuperadas e padronizadas informações como data do nascimento, nome da mãe, endereço de residência para todas as fontes, considerando como referência os dados da fonte Sinan. As inconsistências das variáveis selecionadas foram corrigidas por meio de investigações, tais como busca da completitude dos dados registrados nos bancos disponíveis, ou seja, Sinan, Siscel e SIM, e investigação nas unidades de saúde que notificaram os casos ou digitaram os óbitos. Com os dados completos, as inconsistências corrigidas e a exclusão dos duplicados, foram selecionadas as variáveis identificadoras (nome do paciente, idade, data do nascimento e o nome da mãe) nos bancos de dados Sinan/ Siscel e Sinan/SIM para o relacionamento dos casos no Programa RecLink II.

No programa RecLink II, o processo de blocos de registro (blocking) utilizado inicialmente para emparelhamento dos casos foi constituído pela combinação do último sobrenome e do primeiro nome do paciente, considerando-se o Sinan como fonte de referência e as fontes Siscel e SIM de comparação, ou seja, todos os casos contidos no Siscel e no SIM passaram, um a um, pelo total de casos selecionados no Sinan em 2002 e 2003. Após o cruzamento dos bancos foram identificados os pares verdadeiros, os duvidosos e os não-pares. Procedeu-se à conferência manual dos pares duvidosos com o objetivo de defini-los como verdadeiros ou não-pares, adotandose como critério para classificar como par verdadeiro, os casos em que concordassem todas as variáveis identificadoras ou aqueles que tivessem nomes similares, mesmo apresentando algum problema de grafia, mas nos quais a data do nascimento ou idade e o nome da mãe fossem iguais. Realizados estes procedimentos, foram elaborados no Excel dois novos bancos de dados, Siscel/ Sinan e SIM/Sinan, considerados bancos finais.

Esses bancos foram preparados com o cuidado de definir adequadamente as variáveis para que cada lista de captura pu- 
desse atender às premissas para aplicação do método de captura e recaptura e estimar a subnotificação, quais sejam: 1) a população deve ser fechada, ou seja, durante o período de capturas, o tamanho da população deve ser constante; 2) cada indivíduo capturado deve ser bem marcado, o que facilita a sua identificação para recaptura; 3) as amostras devem ser independentes, ou seja, a possibilidade de um indivíduo estar em uma lista não deve influenciar no fato de estar ou não em outra lista. Quando o método é aplicado na Epidemiologia, entende-se que cada lista utilizada, ou seja, cada fonte de informação é denominada um episódio de captura? .

\section{Análise estatística}

Para estimar a subnotificação dos casos de Aids no município de Fortaleza, nos anos de 2002 e 2003, os métodos de captura e recaptura utilizados foram o estimador de Lincoln-Petersen, obtido pelo método estatístico dos momentos ${ }^{10}$, e o estimador de Chapman, que é um modificador do estimador de Lincoln-Petersen, entre os vários estimadores propostos pela literatura. Os estimadores de Lincoln-Petersen e o de Chapman são construídos sob a suposição de que as amostras nos dois estágios são selecionadas sem reposição ${ }^{11}$. O Estimador de Lincoln-Petersen supõe probabilidade de captura, variando em função das fontes e da independência entre as capturas, sendo definido como:

\section{$\hat{N}=\underline{A} \times \underline{B}$ \\ C}

Onde A e B são os elementos capturados, $\mathrm{C}$ é o número de recapturados e $\mathrm{N}$ o valor desconhecido, o qual se quer estimar. À medida que cresce o número de elementos recapturados, decresce o valor de N. Portanto, quanto maior o número de recapturados, menor é a estimativa do valor real de $\mathrm{N}$.

Este estimador é conhecido como de Lincoln-Petersen ${ }^{12}$. Para que se possa melhor entender o método, observa-se o diagrama de Venn, apresentado na Figura 1.

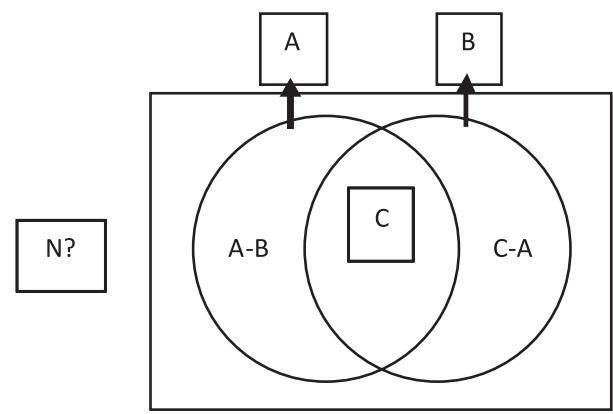

Figura 1 - Diagrama de Venn para duas fontes Figure 1 - Venn diagram for two sources

Uma superestimativa do valor de $\mathrm{N}$ pode ocorrer quando utilizado o estimador de Lincoln-Petersen para amostras que não são grandes. Portanto, torna-se mais apropriado utilizar o estimador de Chapman para a unidade de saúde Hospital Geral de Fortaleza - HGF, permitindo uma aproximação desse estimador e obtendo não tendenciosidade na estimativa de $\mathrm{N}$, uma vez que existe independência entre a captura $\mathrm{e}$ recaptura $^{13}$. A estimativa da subnotificação com intervalo de confiança de IC (95\%) foi realizada utilizando-se o programa Excel Office 2000.

\section{Aspectos éticos}

Esta pesquisa foi submetida ao comitê de ética em pesquisa da Universidade Federal do Ceará e aprovada sob número $308 / 05$.

\section{Resultados}

Foram considerados elegíveis para a pesquisa 1.064 casos de Aids, distribuídos nas três fontes: 688 casos no Sinan; 241 no Siscel e 135 óbitos no SIM. Analisando-se os dados em relação às três fontes, Sinan, Siscel e SIM, observa-se que apenas 10 casos são comuns às três, 151 casos estão no Sinan e Siscel, 106 casos no Sinan e SIM, somente 1 caso no Siscel e SIM . Quanto ao número de casos encontrados isoladamente por fonte, foram identificados 421, 79 e 18 no Sinan, Siscel e SIM, respectivamente (Figura 2). 


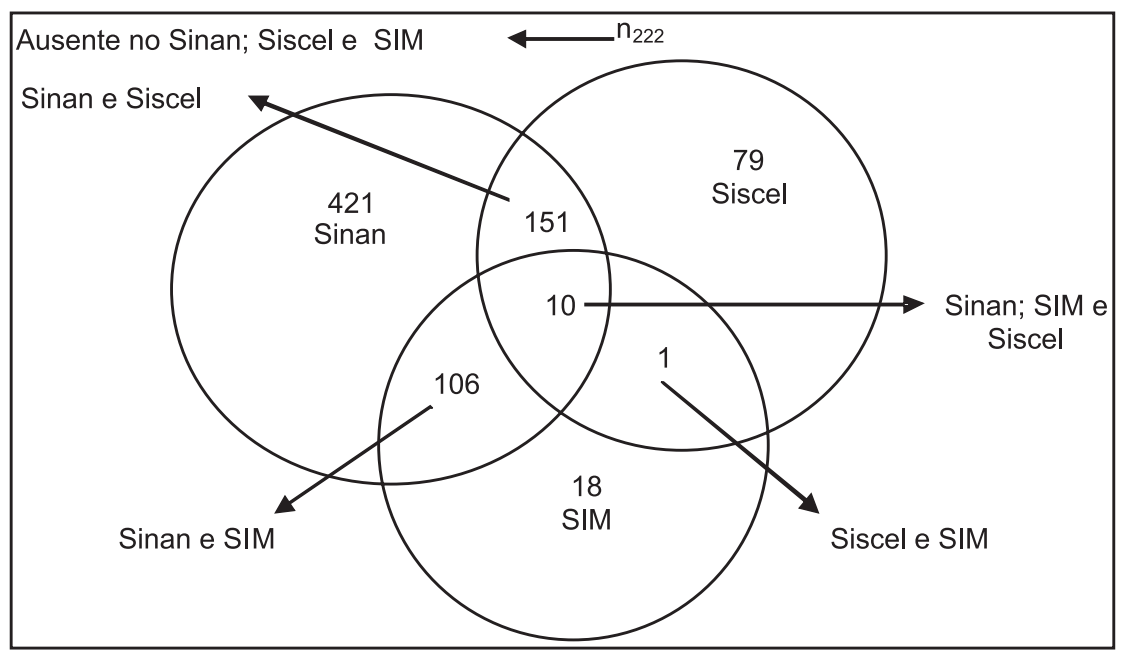

Figura 2 - Diagrama de Venn - Sinan / Siscel e SIM

Figure 2 - Venn diagram - Sinan / Siscel and SIM

Para a análise da subnotificação com a utilização do estimador de Lincoln-Petersen para as fontes Siscel e SIM, foram estimados 1.029 e 801 casos, respectivamente. Para o estimador de Chapman, para as mesmas fontes, os valores estimados foram praticamente iguais, ou seja, 1028 e 800 casos, na mesma ordem. Tendo em vista que o número de casos notificados no Sinan, no período, foi de 688 casos, deixou-se de notificar 341 e 113 casos, respectivamente, para as fontes Siscel eSIM, quando usado o estimador de LincolnPetersen e quando se utilizou o estimador de Chapman o resultado encontrado foi de 340 e 112 casos não notificados. Portanto, não houve praticamente diferença entre os dois estimadores utilizados. A subnotificação no período foi de $33,1 \%$ e 14,1\%, utilizando-se o Siscel e SIM, respectivamente, e tendo como referência o Sinan (Tabela 1).
Considerando a subnotificação para o Hospital São José (668 casos registrados no Sinan e 324 no Siscel), foram estimados 706 casos pelos dois estimadores de Lincoln-Petersen e Chapman. A subnotificação, neste hospital, foi de $5,4 \%$. Já para o HGF (13 casos no Sinan e 81 no Siscel) foram estimados 151 casos, quando o estimador utilizado foi o de Lincoln-Petersen, e 137 casos, quando usado o estimador de Chapman (Tabela 2). Uma superestimativa do valor de $\mathrm{N}$ pode ocorrer quando utilizado o estimador de Lincoln-Petersen para amostras que não são grandes, como no caso do HGF. Portanto, foi considerado o valor estimado de 137 casos, com uma subnotificação de 90,5\% (Tabela 2). Nas demais unidades de saúde onde não foi possível estimar a subnotificação de Aids devido ao número reduzido de casos, calculou-se apenas a taxa de subnotificação,

Tabela 1 - Estimativas pontual e intervalar da subnotificação de casos de Aids no Sinan, baseadas nas fontes Siscel e SIM, conforme os estimadores de Lincoln-Petersen e de Chapman, Fortaleza 2002 e 2003.

Table 1 - Point and interval estimates of underreporting of AIDS in the SINAN based on the Siscel and SIM sources, using the Lincoln-Petersen and Chapman estimators. Fortaleza, 2002-2003.

\begin{tabular}{lcccccc}
\hline Estimador & \multicolumn{3}{c}{ Sinan / Siscel } & \multicolumn{3}{c}{ Sinan / SIM } \\
& N (IC 95\%) & $\mathrm{dp}$ & $\begin{array}{c}\text { Subnotificação } \\
\%(I C ~ 95 \%)\end{array}$ & $\mathrm{N}(\mathrm{IC} 95 \%)$ & $\mathrm{dp}$ & $\begin{array}{c}\text { Subnotificação } \\
\%(I C ~ 95 \%)\end{array}$ \\
\hline Lincoln e Petersen & $1.029(937-1.121)$ & 46,8 & $33,1(30,2-36,0)$ & $801(746-855)$ & 27,9 & $14,1(11,7-16,5)$ \\
\hline Chapman & $1.028(949-1.107)$ & 40,5 & $33,1(30,2-35,9)$ & $800(751-849)$ & 25,1 & $14,0(11,6-16,4)$ \\
\hline
\end{tabular}


Tabela 2 - Estimativas pontual intervalar de subnotificação de casos de Aids no Sinan, baseadas na fonte Siscel por unidade de saúde notificante, utilizando os estimadores de Lincoln-Petersen e de Chapman, Fortaleza 2002 e 2003. Table 2 - Point and interval estimates of underreporting of AIDS cases in the SINAN, based on the Siscel source, by reporting facility using the Lincoln-Petersen and Chapman estimators. Fortaleza 2002-2003.

\begin{tabular}{|c|c|c|c|c|c|c|}
\hline \multirow[t]{2}{*}{ Estimador } & \multicolumn{3}{|c|}{ HSJ* } & \multicolumn{3}{|c|}{$\mathrm{HGF}^{* *}$} \\
\hline & N (IC 95\%) & $d p$ & $\begin{array}{c}\text { Subnotificação } \\
\% \text { (IC 95\%) }\end{array}$ & N (IC 95\%) & $d p$ & $\begin{array}{c}\text { Subnotificação } \\
\% \text { (IC 95\%) }\end{array}$ \\
\hline Lincoln e Petersen & $706(680-732)$ & 13,30 & $5,4(3,7-7,0)$ & $151(24-277)$ & 64,5 & $91,4(86,9-95,9)$ \\
\hline Chapman & $706(683-729)$ & 11,55 & $5,4(3,7-7,0)$ & $137(64-210)$ & 37,3 & $90,5(85,6-95,4)$ \\
\hline
\end{tabular}

que foi de $83,3 \%$ para o Hospital Universitário Walter Cantídio (18 casos registrados no Siscel e apenas 3 constavam no Sinan) e de $100 \%$ para o Hospital Distrital Gonzaga Mota de Messejana (3 casos no Siscel e nenhum notificado no Sinan).

\section{Discussão}

Apesar de todos os esforços envidados para a redução da subnotificação de casos de Aids no Ceará, os resultados deste trabalho apontam para uma importante subnotificação de casos de Aids em adultos, no município de Fortaleza, no período de 2002 e 2003.

Em se tratando das estimativas de subnotificação, objeto deste trabalho, a discussão inicia-se com os métodos empregados. As estimativas foram feitas com a utilização dos estimadores de LincolnPetersen e de Chapman. Observa-se que praticamente não existe diferença entre as estimativas encontradas através dos dois métodos, considerando que a amostra deste estudo é grande. Quando foram utilizados os métodos para a estimação em amostras pequenas, tendo como exemplo a avaliação em uma unidade de saúde com número de casos reduzidos, houve diferença significativa entre os resultados encontrados.

Em relação à taxa de subnotificação avaliada por fonte, os resultados mostram que a subnotificação no Siscel/Sinan foi mais de duas vezes superior, quando comparada à fonte SIM/Sinan. Portanto, destaca-se o Siscel como importante fonte para estudos de subnotificação de Aids. Estudo brasileiro recente relacionou os bancos de dados dos sistemas Sinan, Siscel e Siclom por meio do sistema Reclink ${ }^{7}$. Entretanto, este estudo não estimou a subnotificação através do método captura e recaptura. Outros estudos, embora tenham empregado o método de captura e recaptura, utilizaram outras fontes, como o Sistema de Informações Hospitalares do SUS, Sistema de Informações sobre Mortalidade, prontuários hospitalares, dados das comissões de controle de infecção hospitalar e consultórios médicos ${ }^{8-15}$.

Quanto à taxa de subnotificação encontrada, outros estudos que utilizaram o mesmo método e compararam outras fontes, tiveram resultados diversos. Por exemplo, no estudo realizado em seis municípios brasileiros, ainda que se tenha utilizado o mesmo método e comparado o SIH com o Sinan, a subnotificação encontrada variou de $24 \%$ a $65 \%$ entre os municípios ${ }^{7,15}$. Oliveira $^{15}$, ao estudar a subnotificação de Aids em 1995-96, em Belo Horizonte, utilizando o método de captura e recaptura, encontrou uma subnotificação de $68 \%$. Outro estudo que pode ser citado é o de Buchalla ${ }^{4}$, no qual a taxa de subnotificação em São Paulo, nos anos de 1983-86, resultante da comparação das declarações de óbitos, corresponde ao percentual de $15 \%$ de óbitos não notificados. Em estudo recente, o Estado de São Paulo avaliou a subnotificação anual de óbitos, encontrando um percentual que variou de 5,2\% a 17,0\% de óbitos ainda não notificados no Sinan, considerando os anos de 1980 a $2005^{17}$. Apesar desses estudos 
mostrarem a existência de subnotificação de casos de Aids, fica limitada a comparação dos resultados, tendo em vista a diversidade das fontes de dados estudadas. Mesmo assim, considerando a elevada subnotificação encontrada em Fortaleza, este resultado ainda se encontra abaixo dos achados nos estudos acima citados, com exceção do percentual de $24 \%$ encontrado no município de Florianópolis. Destaca-se que, nos dois últimos estudos citados, avaliou-se apenas a taxa de subnotificação e não a estimativa, através de métodos probabilísticos.

$\mathrm{Na}$ análise da subnotificação por unidade de saúde, as duas principais Unidades de Saúde que atendem pacientes com Aids em Fortaleza, no HSJ e no HGF, apresentaram uma subnotificação de casos de Aids bastante distinta e polarizada. A diferença pode ser atribuída a vários fatores, como a existência de uma Unidade de Vigilância Epidemiológica no HSJ que acumula experiência desde a década de 80 e apresenta uma demanda de atendimento de Aids muito superior às demais unidades notificantes e teve a dispensa de medicamentos para Aids centralizada em suas dependências, durante todo o período de estudo.

Nas outras duas unidades notificantes, Hospital Universitário Walter Cantídio e Hospital Distrital Gonzaga Mota de Messejana, as taxas de subnotificação são preocupantes, haja vista que as unidades hospitalares incluídas nesta pesquisa são de referência para acompanhamento e tratamento de Aids.

\section{Conclusão}

Conclui-se que, apesar dos esforços empreendidos pelos órgãos responsáveis pela vigilância epidemiológica em todas as esferas de governo, ainda existe uma elevada subnotificação dos casos de Aids em Fortaleza. Estes achados indicam que as Secretarias de Saúde do Estado do Ceará e do município de Fortaleza devem empreender esforços para que os profissionais que trabalham com o diagnóstico $\mathrm{e}$ com tratamento da Aids façam a oportuna notificação de todos os casos confirmados. Quanto à vigilância epidemiológica, deve-se buscar a melhoria da qualidade das informações, com a utilização de todas as fontes de dados disponíveis, sobretudo o Siscel, que constitui uma referência útil como fonte de dados para a vigilância da Aids, e também deve ser intensificado o trabalho de emparelhamento dos dados laboratoriais na rotina dos serviços. Por fim, espera-se que esses resultados possam contribuir para a redução da subnotificação dos casos de Aids no município de Fortaleza, o que permitirá um melhor conhecimento da magnitude da epidemia de Aids em Fortaleza e no Ceará.

\section{Referências}

1. Ministério da Saúde (BR). Secretária de Vigilância em Saúde. Guia de vigilância epidemiológica. 6a ed. Brasília; 2005 .

2. Ministério da Saúde (BR). Secretaria de Vigilância em Saúde. Programa Nacional de DST e Aids: critérios de definição de casos de Aids em adultos e crianças. Brasília; 2004a.

3. Barbosa MTS, Struchinern CJ. Estimativas do número de Aids: comparação de métodos que corrigem o atraso da notificação. In: Ministério da Saúde(BR). Coordenação Nacional de DST e AIDS. Simpósio satélite: a epidemia de Aids no Brasil: situação e tendências. Brasília; 1997. p. 15-26.
4. Buchalla CM. A AIDS/SIDA: as estatísticas de mortalidade como fonte de informações. São Paulo: Centro da OMS para Classificação de Doenças em Português; 1990. (Série de Divulgação, n. 6).

5. Ministério da Saúde (BR). Secretaria de Políticas de Saúde. Coordenação de DST e Aids. Vigilância do HIV no Brasil: novas diretrizes. Brasília; 2002.

6. Carvalho DM. Grandes sistemas nacionais de informações em saúde: revisão e discussão da situação atual. Inf Epidemiol SUS 1997; 6(4): 7-46.

7. Lucena FFA, Fonseca MGP, Sousa AA, Coef CM. O relacionamento de bancos de dados na implementação da vigilância da aids. Relacionamento de dados e vigilância da aids. Cad de Saúde Coletiva 2006; 14(2): 305-12, 
8. Ministério da Saúde(BR). A subnotificação de casos de Aids em municípios brasileiros selecionados: uma aplicação do método de captura-recaptura. Bol Epidemiol AIDS 2004b; 18(1): 7- 11.

9. Secretaria da Saúde do Estado (CE). Informe epidemiológico Aids. Fortaleza; 2005.

10. Ferreira VMB, Portela MC, Vasconcelos MTL. Fatores associados à subnotificação de pacientes com Aids, no Rio de Janeiro. Rev Saúde Pública 2000; 34(2): 170-7.

11. Garthwaite PH, Jolliffe IT, Jones B. Statistical inference. New York: Prentice Hall; 1995.

12. Abuabara MAP, Petrele Júnior M. População aberta: o método de Jolly-Seber. In: Abuabara MAP, Petrele Júnior M. Estimativas de abundância de populações animais: introdução às técnicas de captura-recaptura. Paraná: EDUEM; 1997. p. 131-52.

13. LaPorte RE, McCarty DJ, Tull ES. Counting birds, bees and NCDs. Lancet 1992; 339: 494-5.
14. Hook EB, Regal RR. Effect of variation in probability of ascertainment by sources ("variable catchability") upon "capture-recapture" estimates of prevalence. Am J Epidemiol 1993; 137: 1148-66.

15. Oliveira MTC. A subnotificação de casos de Aids em Belo Horizonte, Minas Gerais: uma aplicação da técnica de captura-recaptura [dissertação de mestrado]. Belo Horizonte: Universidade Federal de Minas Gerais; 2000.

16. Buchalla CM. A Síndrome de Imunodeficiência Adquirida e a mortalidade masculina de 20 a 49 anos, município de São Paulo, 1983-1986 [tese de doutorado]. São Paulo: Faculdade de Saúde Pública da Universidade de São Paulo; 1993.

17. Secretaria de Estado da Saúde (SP). A Vigilância Epidemiológica da Aids no Estado de São Paulo (dados até 30/06/2005), Bol Epidemiol C.R.T. DST/Aids C.V.E. 2005; 24(1): 25.

Recebido em: 09/05/07

Versão final reapresentada em: 28/05/08

Aprovado em: 30/05/08 